\title{
1.5 Tesla Non-ultrashort but Short Echo Time Magnetic Resonance Angiography Describes the Arteries Near a Clipped Cerebral Aneurysm
}

\author{
Yoichi Higo $^{1}$, Sakura Komagata ${ }^{1}$, Masahito Katsuki ${ }^{2}$, Shin Kawamura ${ }^{2}$, Akihito Koh ${ }^{2}$ \\ 1. Department of Radiological Technology, Itoigawa General Hospital, Itoigawa, JPN 2. Department of Neurosurgery, \\ Itoigawa General Hospital, Itoigawa, JPN
}

Corresponding author: Masahito Katsuki, ktk1122nigt@gmail.com

\begin{abstract}
Cerebral aneurysm and mother artery assessment after clipping is essential to evaluate aneurysm remnant, regrowth, and clip slippage. Usually, cerebral angiography and contrast-enhanced computed tomography angiography (CTA) are used for the evaluation, but they have the side effect of contrast medium and are time-consuming. Time-of-flight magnetic resonance angiography (TOF-MRA) is a non-invasive and fast modality, but clip-induced artifacts limit the signal near the metal clip. Recent ultrashort echo time (UTE)MRA reduces metal artifacts but its availability is still low worldwide. Therefore, we developed a modified TOF-MRA sequence, named short TE-MRA, using Optima MR 360 1.5T Advance (GE Healthcare Life Sciences, Buckinghamshire, UK). It could describe the artery near the clip using general MRA equipment without recent UTE-MRA technology. We present a subarachnoid hemorrhage patient who underwent short TE-MRA about a year after clipping for the aneurysms at the bilateral internal carotid arteries. Short TE-MRA described the left internal carotid, middle cerebral, and anterior cerebral arteries. The right middle and anterior cerebral arteries were described, but the right internal carotid artery was not. Normal TOF-MRA could not describe them. Without recent UTE-MRA technology, short TE-MRA might be an alternative method for evaluating the artery near the clip. Short TE-MRA can be performed by general MRA equipment with a little time, so it may be helpful until UTE-MRA is widely used. Further research is needed on whether short TE-MRA can describe the aneurysm remnant, regrowth, and clip slippage up to the clinically useful level.
\end{abstract}

Categories: Neurology, Radiology, Neurosurgery

Keywords: cerebral aneurysm, clipping, time-of-flight magnetic resonance angiography (tof-mra), ultrashort echo time magnetic resonance angiography (ute-mra), less invasive, magnetic resonance imaging, clip artifact

\section{Introduction}

The cerebral aneurysm and mother artery assessment after clipping is essential to evaluate aneurysm remnant, regrowth, clip slippage, and kink of the arteries. Usually, cerebral angiography (CAG) and contrastenhanced computed tomography angiography (CTA) are used for the evaluation [1], but they have side effects of contrast medium and are time-consuming. Of course, less invasive methods are better for the aneurysm and artery assessment. Time-of-flight magnetic resonance angiography (TOF-MRA) is a noninvasive and fast modality, but clip-induced metal artifact limits the signal near the clip [2]. Recent ultrashort echo time (UTE)-MRA reduces metal artifact [3-11]. However, UTE-MRA is a recent sequence and costs much, so its availability is still low worldwide. Therefore, we tried to make a new sequence, which describes the artery near the metal clip without the latest MRA equipment. We developed a modified TOFMRA sequence, named short TE-MRA, using Optima MR 360 1.5T Advance (GE Healthcare Life Sciences, Buckinghamshire, UK). Short TE-MRA could describe the artery near the clip without recent UTE-MRA technology nor other modalities using contrast medium.

\section{Case Presentation}

An 80-year-old woman developed subarachnoid hemorrhage (SAH), Hunt and Kosnik grade II, due to a ruptured aneurysm at the left internal carotid artery (ICA). She also had an unruptured aneurysm at the right ICA. TOF-MRA five years before the onset revealed the $7 \mathrm{~mm}$ aneurysm at the left ICA (red arrowheads, Figure 1, panel A) and the $5 \mathrm{~mm}$ aneurysm at the right ICA (blue arrowheads, Figure 1, panel A). We performed first left and then right frontotemporal craniotomies and clipping using the fourth-generation YASARGIL titanium clips \#760 (Aesculap, Tuttlingen, Germany) as a two-stage surgery in three days, just in case of misidentifying the ruptured aneurysm [12]. The clips were made of TiAl6V4 titanium alloy (ISO 5832-2). On the three-postoperative day after the second surgery, CTA revealed the clips and bilateral ICAs, and complete clipping was confirmed (thick red and blue arrows, Figure 1, panel B). The patient was discharged home after a two-month rehabilitation with a modified Rankin Scale 4. 


\section{Cureus}

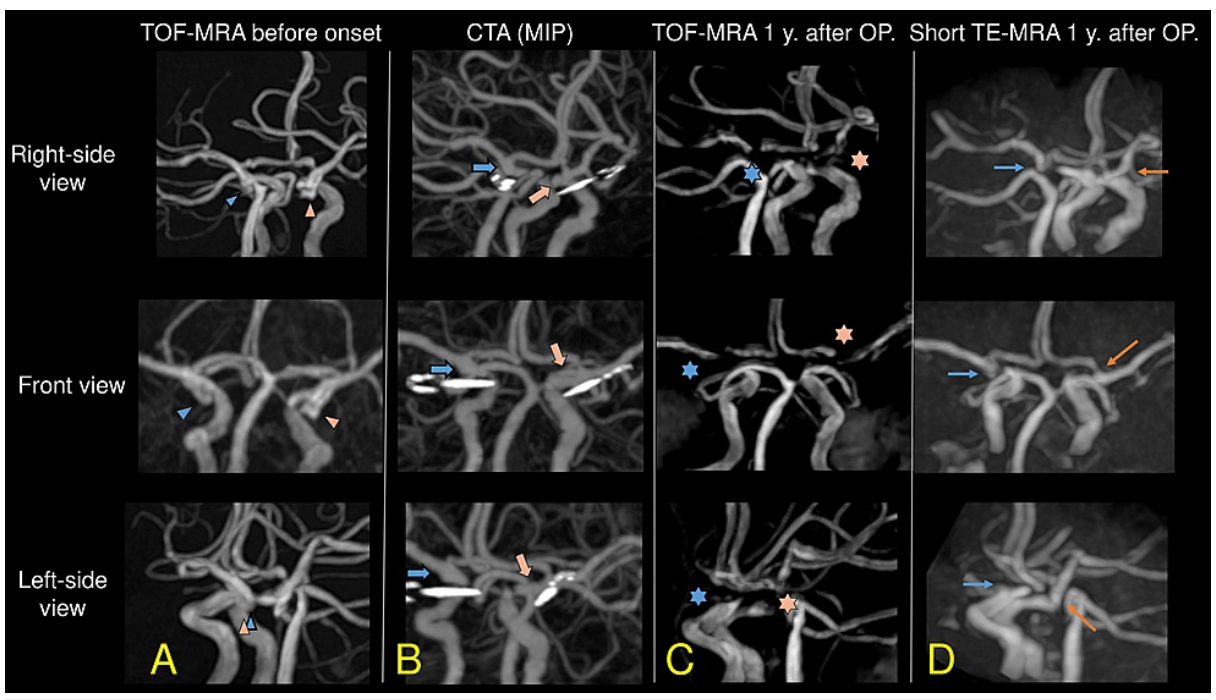

\section{FIGURE 1: Comparison of each MRA sequence}

TOF-MRA before the subarachnoid hemorrhage onset revealed the $7 \mathrm{~mm}$ aneurysm at the left ICA (red arrowheads, A) and the $5 \mathrm{~mm}$ aneurysm at the right ICA (blue arrowheads; A). On the third postoperative day after clipping, CTA revealed the clips and bilateral ICAs (thick red and blue arrows; B). About a year after the clipping, 1.5T TOF-MRA did not describe the bilateral ICAs, nor some parts of MCAs and ACAs (red and blue asterisks; C). Short TE-MRA did not describe the right ICA (thin blue arrows, D), but it revealed the right MCA and $\mathrm{A} 1$ portion of the ACA. Also, it revealed those on the left (thin red arrows, D).

Abbreviations in the figures: CTA; computed tomography angiography, OP; operation, MIP; maximum intensity projection, TE; echo time TOF-MRA; time-of-flight magnetic resonance angiography

About a year after the surgery, we performed TOF-MRA and short TE-MRA to evaluate the bilateral arteries near the clip. 1.5T TOF-MRA did not describe the bilateral ICAs. Furthermore, some parts of the bilateral middle cerebral arteries (MCAs) and A1 portions of the anterior cerebral arteries (ACAs) were not described (red and blue asterisks, Figure 1, panel C). Short TE-MRA did not describe the right ICA, but it revealed the right MCA and A1 portion of the ACA (thin blue arrows, Figure 1, panel D). Short TE-MRA revealed those on the left (thin red arrows, Figure 1, panel D). The bilateral posterior communicating arteries were not described in both CTA and short TE-MRA due to their narrow diameters and the metal artifacts of the clips.

We acquired the CTA using an 80-row CT scanner (Aquillion Prime SP; Canon Medical Systems, Tochigi, Japan). Scan parameters comprised rotation period, $0.5 \mathrm{sec}$; tube voltage, $20 \mathrm{kV}$; tube current, 150-350 mA; slice thickness, $0.5 \mathrm{~mm}$; matrix, $512 \times 512$; and field of view, $200 \mathrm{~mm}$. Iopamidol was used as a contrast medium. The maximum intensity projection images were made using Aquarius iNtuition Client Viewer (TeraRecon Incorporated, Durham).

To acquire the TOF-MRA and short TE-MRA, we used Optima MR 360 1.5T Advance with an 8-channel headneck coil. The detailed scan parameters for TOF-MRA: repetition time/echo time, 32/6.8 ms; flip angle, 18 degrees; field of view, $200 \times 200 \mathrm{~mm}$; matrix, $288 \times 192$; thickness, $1.2 \mathrm{~mm}$; and acquisition time, 301 seconds. Those for short TE-MRA: repetition time/echo time, $32 / 1.2 \mathrm{~ms}$; flip angle, 18 degrees; field of view, $220 \times 220 \mathrm{~mm}$; matrix, $160 \times 160$; thickness, $1.6 \mathrm{~mm}$; and acquisition time, 173 seconds. The images of TOFand short TE-MRA were acquired as axial images.

Our hospital's research ethics committee approved this study (ethical approval number 2021-4). We gained written informed consent for this study from the patients. All methods were carried out under relevant guidelines and regulations (Declaration of Helsinki).

\section{Discussion}

This is the first report on the clinical usefulness of modified TOF-MRA, named short TE-MRA, for postclipping assessment on the cerebral arteries. Compared to CTA, short TE-MRA is less invasive and fast, but its image quality is not up to the CTA. The description range and the image quality of short TE-MRA were inferior to TOF-MRA, but short-TE MRA could describe some of the mother vessels. Compared to UTE-MRA, short TE-MRA does not need recent special equipment for UTE-MRA [13], so short TE-MRA is available using standard equipment worldwide. Furthermore, the acquisition time for UTE-MRA is long as five to 10 minutes due to multiple scans and subtraction processing [13]. In contrast, the acquisition time for short TEMRA is only 173 seconds, so it is not a labor-intensive and time-consuming task to be incorporated into daily magnetic resonance imaging scans. 
We hypothesize that UTE-MRA, such as pointwise encoding time reduction with radial acquisition (PETRA)MRA (Siemens, Germany) [8] and SILENT MRA (GE Healthcare Life Sciences, UK) [9], will be the gold standard to evaluate the aneurysms and arteries after clipping in the future as to CAG and CTA. However, they have not yet been widespread. Of course, CAG and CTA are superior to both UTE-MRA and short TEMRA for the description of the arteries near the clip [14]. However, short TE-MRA is less invasive and not time-consuming so it may be helpful for routine outpatient follow-up for the patients after clipping instead of frequent CAG or CTA.

\section{Conclusions}

Short TE-MRA could be performed by general MRA equipment, and it takes a little time. We performed the sequence without the latest MRA equipment. It described an artery that the usual TOF-MRA could not show. Without a recent UTE-MRA sequence, short TE-MRA might be an alternative method for evaluating the artery near the clip. Further research is needed on whether short TE-MRA can describe the aneurysm remnant, regrowth, and clip slippage. Also, it should be clarified what kind of aneurysm, clip shape, and surgical procedure, like how to apply the clip, can be applied to short TE-MRA.

\section{Additional Information \\ Disclosures}

Human subjects: Consent was obtained or waived by all participants in this study. Itoigawa General Hospital Ethics Committee issued approval 2021-4. Our hospital's research ethics committee approved this study (ethical approval number 2021-4). We gained written informed consent for this study from the patients. All methods were carried out under relevant guidelines and regulations (Declaration of Helsinki). Conflicts of interest: In compliance with the ICMJE uniform disclosure form, all authors declare the following: Payment/services info: All authors have declared that no financial support was received from any organization for the submitted work. Financial relationships: All authors have declared that they have no financial relationships at present or within the previous three years with any organizations that might have an interest in the submitted work. Other relationships: All authors have declared that there are no other relationships or activities that could appear to have influenced the submitted work.

\section{Acknowledgements}

Authors YH and SK have equally contributed to this article.

\section{References}

1. van Loon JJ, Yousry TA, Fink U, Seelos KC, Reulen HJ, Steiger HJ: Postoperative spiral computed tomography and magnetic resonance angiography after aneurysm clipping with titanium clips. Neurosurgery. 1997, 41:851-6; discussion 856-7. 10.1097/00006123-199710000-00016

2. Becker RL, Norfray JF, Teitelbaum GP, Bradley WG Jr, Jacobs JB, Wacaser L, Rieman RL: MR imaging in patients with intracranial aneurysm clips. AJNR Am J Neuroradiol. 1988, 9:885-9.

3. Koch KM, Hargreaves BA, Pauly KB, Chen W, Gold GE, King KF: Magnetic resonance imaging near metal implants. J Magn Reson Imaging. 2010, 32:773-87. 10.1002/jmri.22313

4. Gönner F, Lövblad KO, Heid O, Remonda L, Guzman R, Barth A, Schroth G: Magnetic resonance angiography with ultrashort echo times reduces the artefact of aneurysm clips. Neuroradiology. 2002, 44:755-8. 10.1007/s00234-002-0825-8

5. Schmalbrock P, Yuan C, Chakeres DW, Kohli J, Pelc NJ: Volume MR angiography: methods to achieve very short echo times. Radiology. 1990, 175:861-5. 10.1148/radiology.175.3.2343137

6. Robson MD, Gatehouse PD, Bydder M, Bydder GM: Magnetic resonance: an introduction to ultrashort TE (UTE) imaging. J Comput Assist Tomogr. 2003, 27:825-46. 10.1097/00004728-200311000-00001

7. Takubo S, Kawasaki K, Nagatari T, Matsumoto M, Kageyama T: Clinical usefulness of ultra-short TE MRA for follow-up imaging after cerebral aneurysm clipping [ Article in Japanese]. Nihon Hoshasen Gijutsu Gakkai Zasshi. 2020, 76:177-84. 10.6009/jirt.2020 JSRT 76.2.177

8. Nishikawa A, Kakizawa Y, Wada N, Yamamoto Y, Katsuki M, Uchiyama T: Usefulness of pointwise encoding time reduction with radial acquisition and subtraction-based magnetic resonance angiography after cerebral aneurysm clipping. World Neurosurg X. 2021, 9:100096. 10.1016/j.wnsx.2020.100096

9. Katsuki M, Narita N, Ishida N, et al.: Usefulness of 3 Tesla ultrashort echo time magnetic resonance angiography (UTE-MRA, SILENT-MRA) for evaluation of the mother vessel after cerebral aneurysm clipping: Case series of 19 patients. Neurol Med Chir (Tokyo). 2021, 61:193-203. 10.2176/nmc.oa.2020-0336

10. Katsuki M, Narita N, Ozaki D, Sato Y, Iwata S, Tominaga T: Three tesla magnetic resonance angiography with ultrashort echo time describes the arteries near the cerebral aneurysm with clip and the peripheral cerebral arteries. Surg Neurol Int. 2020, 11:224. 10.25259/SNI_329_2020

11. Katsuki M, Kakizawa Y, Yamamoto Y, Nishikawa A, Wada N, Uchiyama T: Magnetic resonance angiography with ultrashort echo time evaluates cerebral aneurysm with clip. Surg Neurol Int. 2020, 11:65. 10.25259/SNI_59_2020

12. Katsuki M, Wada N, Yamamoto Y: Single-stage clipping with bifrontal and bilateral frontotemporal craniotomies for subarachnoid hemorrhage with multiple cerebral aneurysms using Sugita head holding system: A case report. Surg Neurol Int. 2020, 11:76. 10.25259/SNI 732020

13. Kiriki M, Jomoto W, Ikeda T, Kotoura N: Imaging parameter optimization of 3D phase contrast-MRA to reduce susceptibility-artifact and radiofrequency-shielding around the intracranial stent [Article in 


\section{Cureus}

Japanese]. Nihon Hoshasen Gijutsu Gakkai Zasshi. 2018, 74:1293-301. 10.6009/jjrt.2018_JSRT_74.11.1293

14. Ryu KH, Baek HJ, Moon JI, et al.: Usefulness of noncontrast-enhanced silent magnetic resonance

angiography (MRA) for treated intracranial aneurysm follow-up in comparison with time-of-flight MRA.

Neurosurgery. 2020, 87:220-8. 10.1093/neuros/nyz421 